\title{
Use of autoantigen-knockout mice in developing an active autoimmune disease model for pemphigus
}

\author{
Masayuki Amagai, ${ }^{1}$ Kazuyuki Tsunoda, ${ }^{1}$ Harumi Suzuki, ${ }^{2}$ Koji Nishifuji, ${ }^{1}$ \\ Shigeo Koyasu, ${ }^{2}$ and Takeji Nishikawa ${ }^{1}$ \\ ${ }^{1}$ Department of Dermatology, and \\ ${ }^{2}$ Department of Immunology, Keio University School of Medicine, 35 Shinanomachi, Shinjuku-ku, Tokyo 160-8582, Japan \\ Address correspondence to: Masayuki Amagai, Department of Dermatology, Keio University School of Medicine, \\ 35 Shinanomachi, Shinjuku-ku, Tokyo 160-8582, Japan. \\ Phone: 81-3-3353-1211 ext.62414; Fax: 81-3-3351-6880; E-mail: amagai@mc.med.keio.ac.jp. \\ Or to: Shigeo Koyasu, Department of Immunology, Keio University School of Medicine, 35 Shinanomachi, Shinjuku-ku, \\ Toyko 160-8582, Japan. Phone: 81-3-5363-3768; Fax: 81-3-5361-7658; E-mail: koyasu@sun.microb.med.keio.ac.jp.
}

Received for publication October 21, 1999, and accepted in revised form January 26, 2000.

The development of experimental models of active autoimmune diseases can be difficult due to tolerance of autoantigens, but knockout mice, which fail to acquire tolerance to the defective gene product, provide a useful tool for this purpose. Using knockout mice lacking desmoglein 3 (Dsg3), the target antigen of pemphigus vulgaris (PV), we have generated an active disease model for this autoantibody-mediated disease. $D s g 3^{-/-}$mice, but not $D s g 3^{+/-}$littermates, produced anti-Dsg3 $\mathrm{IgG}$ that binds native Dsg3, when immunized with recombinant mouse Dsg3. Splenocytes from the immunized Dsg $3^{-/-}$mice were then adoptively transferred into Rag-2-/- immunodeficient mice expressing Dsg3. Anti-Dsg3 IgG was stably produced in the recipient mice for more than 6 months without further boosting. This IgG bound to Dsg3 in vivo and disrupted the cell-cell adhesion of keratinocytes. Consequently, the recipient mice developed erosions in their oral mucous membranes with typical histologic findings of PV. In addition, the recipient mice showed telogen hair loss, as found in $\mathrm{Dsg} 3^{-/}$mice. Collectively, the recipient mice developed the phenotype of PV due to the pathogenic anti-Dsg3 IgG. This model will be valuable for developing novel therapeutic strategies. Furthermore, our approach can be applied broadly for the development of various autoimmune disease models.

J. Clin. Invest. 105:625-631 (2000).

\section{Introduction}

Self-tolerance is acquired as a result of clonal deletion or the inactivation of developing lymphocytes that are potentially harmful to the body (1-3). This prevents the immune system from reacting destructively against self components, which can lead to devastating autoimmune diseases. On the other side of the same coin, however, it is very difficult to develop experimental models for autoimmune diseases, which are pivotal for dissecting the mechanisms of tolerance and autoimmunity, as well as for developing novel therapeutic strategies. In this study, we attempted to overcome this difficulty by using autoantigen-knockout mice. In these mice, selftolerance of the defective gene product is not acquired because lymphocytes are never exposed to the target antigen during development. Adoptive transfer of lymphocytes from autoantigen-knockout mice after immunization with the antigen, into mice expressing the antigen, should generate an autoimmune reaction in the recipient mice, thus providing an active disease model for autoimmune disease. To test this hypothesis, we used a well-defined autoimmune disease against skin and mucous membranes, pemphigus vulgaris (PV).

$\mathrm{PV}$ is a life-threatening autoimmune disease of the skin and mucous membranes that is histologically characterized by blister formation due to the loss of cell-cell adhesion of keratinocytes, and immunopathologically by the presence of in vivo bound and circulating IgG directed against the cell surface of keratinocytes in vivo (4). Clinically, patients with PV develop widespread flaccid blisters and painful erosions, which can occur in any stratified squamous epithelium. The target antigen of $\mathrm{PV}$, desmoglein 3 (Dsg3), is a transmembrane desmosomal protein that belongs to the cadherin supergene family of cell-cell adhesion molecules (5-7). Compelling evidence has accumulated for the pathogenicity of $\operatorname{IgG}$ autoantibodies against Dsg3 in PV (8-12).

In this study, we developed an active autoimmune disease model of PV using mice that are genetically deficient in the target antigen for PV. We immunized Dsg $3^{-/-}$mice (13) with mouse recombinant Dsg3 (rDsg3), and then adoptively transferred their splenocytes into Rag $2^{-1-}$ immunodeficient mice that express Dsg3. The recipient mice stably produced the pathogenic anti-Dsg3 IgG and exhibited the phenotype of PV. Our approach can be widely applied in developing experimental models of various autoimmune diseases.

\section{Methods}

Construction of recombinant mouse Dsg3 and Dsg1 protein. A cDNA encoding the entire extracellular domain of mouse Dsg3 (GenBank U86016) was PCR amplified on 
a phage clone containing mouse Dsg3 cDNA as a template (a kind gift from Jouni Uitto, Jefferson Medical College, Philadelphia, Pennsylvania, USA) with the appropriate primers (5'-CCGAGATCTCCTATAAATATGACCTGCCTCTTCCCTAGA-3' and 5'-CGGGTCGACCCTCCAGGATGACTCCCCATA-3'). In the same way, a cDNA encoding the entire extracellular domain of mouse Dsg1, the autoantigen of pemphigus foliaceus, was PCR amplified on a plasmid clone containing mouse Dsg1 cDNA (a kind gift from Norihisa Matsuyoshi, and John R. Stanley, University of Pennsylvania; and Leena Pulkinen, and Jouni Uitto, Jefferson Medical College) with another pair of primers (5'-CCGAGATCTCCTATAAATATGGACTGGCACTCCTTCAGG-3' and 5'-CGGCTCGAGGTGAACGTTGTCTCCATAGAG-3'). These cDNAs were subcloned into $\mathrm{pEVmod-Dsg3-His} \mathrm{vector}$ (14) in place of cDNA for human Dsg3 (pEVmodmDsg3-His, pEVmod-mDsg1-His). Recombinant baculoproteins, mouse rDsg3 and rDsg1, were prepared as previously described $(15,16)$.

Mice. Dsg3 $3^{-/}$mice were obtained by mating male Dsg $3^{-/-}$mice and female $D s g 3^{+/-}$mice (The Jackson Laboratory, Bar Harbor, Maine, USA) (13). Dsg $3^{-/-}$mice have a mixed genetic background of $129 / \mathrm{SV}\left(\mathrm{H}-2^{\mathrm{b}}\right)$ and C57BL/6J (H-2 $)$ (13). Rag-2-/- mice that had been backcrossed to B6.SJL-Ptprc ${ }^{a}$ mice for 10 generations were obtained from Taconic Farms (Germantown, New York, USA) (17).

ELISA. Circulating anti-Dsg3 IgG was measured by ELISA using mouse rDsg3 as a coated antigen as previously described $(14,18)$. Each sample was diluted 50fold and run in duplicate. A single serum sample obtained from a $\mathrm{sg}^{-/-}$mouse immunized with mouse rDsg3 was used as a positive control, and serum from a nonimmunized mouse was used as a negative control. ELISA scores were obtained as index values with the following formula: index value $=\left(\mathrm{OD}_{450}\right.$ of sample $-\mathrm{OD}_{450}$ of negative control $) /\left(\mathrm{OD}_{450}\right.$ of positive control - $\mathrm{OD}_{450}$ of negative control) $\times 100$ (ref. 18). When the OD exceeded 2.0, the serum sample was further diluted and the index value was multiplied by the dilution factor. The ELISA scores against mouse rDsg1 were measured in the same way using rDsg1-coated ELISA plates.

Living keratinocyte staining. A mouse keratinocyte cell line, PAM212 (19), was incubated with mouse serum samples diluted 20-fold with DMEM containing 10\% FCS at $37^{\circ} \mathrm{C}$ in a $\mathrm{CO}_{2}$ incubator for 30 minutes. After being washed with PBS, the cells were fixed with $100 \%$ methanol at $-20^{\circ} \mathrm{C}$ for 20 minutes, and incubated with FITC-conjugated goat anti-mouse antibodies (DAKO A/S, Glostrup, Denmark) at room temperature for 30 minutes. Specimens were examined under an Eclipse E800 fluorescent microscope (Nikon Corp. Tokyo, Japan).

Immunization of mice. Mice were primed by intraperitoneal injection of $5 \mu \mathrm{g}$ of purified mouse rDsg3 in complete Freund's adjuvant on day 0 . They were subsequently boosted with mouse rDsg3 in incomplete Freund's adjuvant twice, and then injected with mouse
rDsg3 without adjuvant twice each week. Antibody production was examined by ELISA at the indicated time.

Adoptive transfer of splenocytes. Splenocytes were isolated from $\mathrm{Dsg}^{-/-}$or $\mathrm{Dsg3}^{+/-}$mice on day 32 (4 days after boosting with mouse rDsg3 without adjuvant at day 28). Typically, the splenocytes were pooled from $2 \mathrm{immu-}$ nized $D s g 3^{-/-}$or $D s g 3^{+/-}$mice and then administered to 10 $R a g-2^{-1-}$ mice. Intravenous injection into the tail vein of Rag- $2^{-/-}$mice was used to transfer $10^{7}$ splenocytes in 500 $\mu \mathrm{L}$ PBS per mouse.

ELISPOT assay. PVDF-bottomed 96-well Amicon multititer plates (Millipore Corp., Beverly, Massachusetts, USA) were coated with $30 \mu \mathrm{g} / \mathrm{mL}$ of mouse rDsg3. Mononuclear cells prepared from the peripheral blood, spleen, bone marrow, and lymph nodes of reconstructed $\mathrm{Rag}-2^{-/-}$mice were incubated on the plates at $37^{\circ} \mathrm{C}$ in a $\mathrm{CO}_{2}$ incubator for 4 hours. IgG bound to the membrane was revealed as spots with alkaline phosphatase-conjugated anti-mouse IgG antibodies (Zymed Laboratories Inc., South San Francisco, California, USA). The number of spots was counted under a dissecting microscope, and the frequency of antiDsg3 IgG-producing B cells was defined as the number of spots in $10^{5}$ mononuclear cells. All experiments were carried out in triplicate.

\section{Results}

Dsg3 $3^{-/}$mice, but not wild-type mice, produced anti-Dsg3 IgG capable of binding to the native mouse Dsg3 on living keratinocytes. Initially, we tested various wild-type mice (C57BL/6N, BALB/c, and C3H/HeJ) for their ability to produce anti-Dsg3 IgG upon immunization. However, none of them produced IgG that was able to recognize the native form of mouse Dsg3 (data not shown). We believe this failure was probably due to immunologic tolerance against mouse Dsg3, a self-antigen.

Such tolerance should not be acquired in mice that are genetically deficient in Dsg3. When we immunized Dsg $3^{-/-}$mice with mouse rDsg3, anti-Dsg3 IgG was indeed produced. The ELISA titers against rDsg3 became positive at day 11 in $\mathrm{Dsg}^{-/-}$mice, and their titers continued to increase thereafter (Figure 1a). These sera were able to bind to the cell surfaces of living cultured mouse keratinocytes (Figure 1b, left), indicating that the anti-Dsg3 IgG produced in $D s g 3^{-/-}$mice is capable of binding to the native Dsg3 on living keratinocytes. In contrast, although the ELISA titers of Dsg $3^{+/-}$littermates became positive after repeated immunization, their titers were lower than those of Dsg $3^{-/-}$mice (day 33, $P=0.0016$ ). More importantly, the sera from $D s g 3^{+/-}$mice failed to bind to the surface of living keratinocytes (Figure 1b, right). In addition, there was no in vivo deposition of IgG in the stratified squamous epithelia of the immunized $\mathrm{Dsg}^{+/-}$mice (data not shown). Therefore, most of the antibodies developed in $D s g 3^{+/-}$mice may have been raised against minor contaminants of the recombinant protein preparation, tags introduced at the $\mathrm{COOH}$-terminus of rDsg3, or degraded products. These findings indicate 


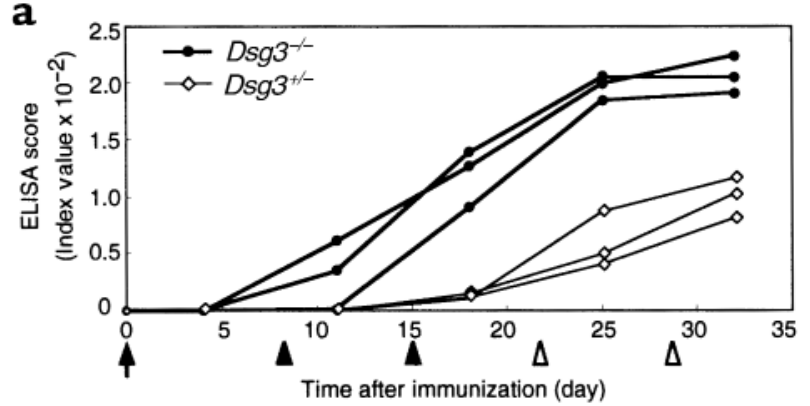

b

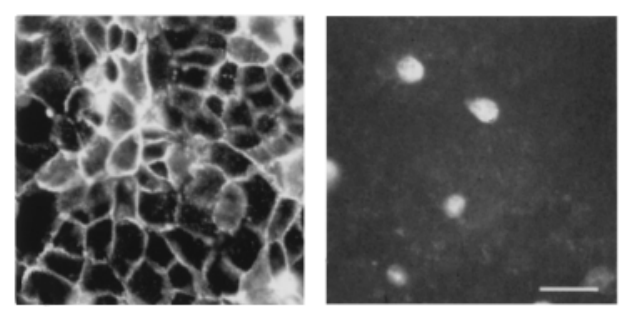

\section{Figure 1}

Anti-Dsg3 IgG antibodies that can bind the native Dsg3 in vivo are produced in Dsg3-/- mice, but not in $D s g 3^{+/-}$mice. (a) $D s g 3^{-/-}$mice and their $D s 3^{+/-}$littermates were immunized with mouse rDsg3, and the ELISA titers against rDsg3 were measured over time. Mice were primed by intraperitoneal injection of purified mouse rDsg3 in complete Freund's adjuvant on day 0 (arrow). They were subsequently boosted with mouse rDsg3 in incomplete Freund's adjuvant (solid triangles), and then injected with the mouse rDsg3 without adjuvant (open triangles). (b) A mouse keratinocyte cell line, PAM212, was incubated with mouse serum samples in culture media in a $\mathrm{CO}_{2}$ incubator for 30 minutes. After being washed and fixed with methanol, bound mouse IgG was revealed with FITC-conjugated goat antimouse IgG antibodies. Sera from immunized $D s g 3^{-1-}$ mice (left), but not from their $D s g 3^{+/-}$littermates (right), stained the cell-cell contact sites of cultured keratinocytes. Bar, $50 \mu \mathrm{m}$.

that $D s g 3^{-/-}$mice and mice expressing Dsg3 have a clear difference in their ability to produce anti-Dsg3 IgG that can bind to native Dsg3.

Stable production of anti-Dsg3 IgG by lymphocytes of immu-

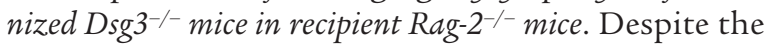
production of anti-Dsg3 IgG, no autoimmune reaction was expected in the immunized $D s g 3^{-/-}$mice because these mice lack the target antigen. To allow the antiDsg3 IgG to be exposed to the antigen, we isolated splenocytes from the immunized $D s g 3^{-/-}$mice, or from $D s g 3^{+/-}$mice as controls, and transferred them into Rag$2^{-/-}$immunodeficient mice (17) that do express Dsg3.
Rag-2-/- mice have no mature $\mathrm{T}$ or $\mathrm{B}$ cells, due to their inability to rearrange $\mathrm{T}$-cell receptor or immunoglobulin genes, and thus are unable to produce antibodies or to reject the transferred splenocytes.

Circulating anti-Dsg3 IgG was detected in the sera of recipient Rag- $2^{-/-}$mice as early as day 4 after the transfer of $D s g 3^{-/-}$splenocytes. The level increased rapidly without further boosting by rDsg3, and reached a plateau around day 21 (Figure 2a). The circulating anti-Dsg3 IgG was detected for as long as these mice were assayed, which was more than 6 months, indicating that endogenous Dsg3 in the recipient mice stimulated the transferred Dsg3-specific lymphocytes from the immunized $D s g 3^{-/-}$mice in vivo. Furthermore, the ELISPOT assay also detected anti-Dsg3 IgG-producing B cells in the spleen and lymph nodes of the recipient mice 4 months after the adoptive transfer (Table 1), confirming the persistent ongoing antibody production. No significant reactivity against Dsg1, another desmosomal cadherin targeted in pemphigus foliaceus, was observed in these recipient mice during this period (Figure 2a). In marked contrast, no circulating anti-Dsg3 IgG was detected in

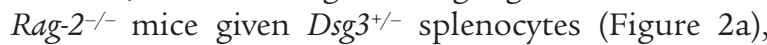
although the numbers of $\mathrm{CD} 4^{+} \mathrm{T}$ cells and $\mathrm{CD} 19^{+} \mathrm{B}$ cells from $D s g 3^{+/-}$mice were comparable with those from $D s g 3^{-/-}$mice in the recipient mice (data not shown).

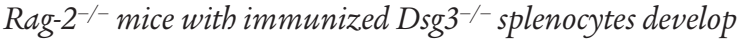
$P V$ phenotype. The first symptom we noticed in the recipient $R a g-2^{-1-}$ mice that received immunized $D s g 3^{-/-}$ splenocytes was weight loss that began between day 7 and day 14 after the adoptive transfer (Figures $2 b$ and $3 a$, Table 2). In the following days, these mice continued to lose weight, and some of them died. The mice that survived started to gain weight again about day 35 . Some of these recipient Rag-2-/- mice developed crusted erosions on the skin around the snout (Figure 3b), an area that is normally traumatized by scratching.

In $R a g-2^{-/-}$mice that received immunized $\mathrm{Dsg}^{-/-}$ splenocytes, in vivo $\operatorname{IgG}$ deposition was found on keratinocyte cell surfaces in stratified squamous epithelium, including the skin (Figure $3 c$, the skin around the snout), and oral (Figure 3d, hard palate) and esophageal mucous membranes, just as is seen in patients with PV (Figure 3f). In the epidermis where there were multiple layers of keratinocytes, IgG deposition was limited to the lower layers, whereas in the oral and esophageal epithelium, IgG was found

Table 1

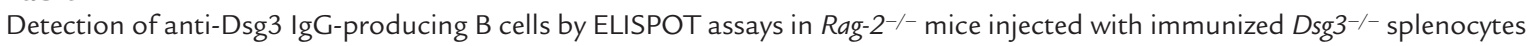

\begin{tabular}{lcccccr}
\cline { 2 - 3 } & Transfer & Day $^{\mathrm{A}}$ & Spleen & Lymph node & Bone marrow & PBMC \\
$\# 466$ & - & - & $0.0 \pm 0.0$ & $0.0 \pm 0.0$ & $0.0 \pm 0.0$ & $0.0 \pm 0.0$ \\
$\# 514$ & + & 22 & $102.1 \pm 14.7$ & $13.5 \pm 13.6$ & $0.0 \pm 0.0$ & $3.8 \pm 5.4$ \\
$\# 212$ & + & 33 & $20.8 \pm 5.9$ & $16.8 \pm 8.8$ & $0.0 \pm 0.0$ & $0.0 \pm 0.0$ \\
$\# 134$ & + & 117 & $31.3 \pm 8.8$ & $27.1 \pm 2.9$ & $0.1 \pm 2.9$ & $0.0 \pm 0.0$ \\
$\# 135$ & + & 117 & & & $0.0 \pm 0.0$ \\
\hline
\end{tabular}

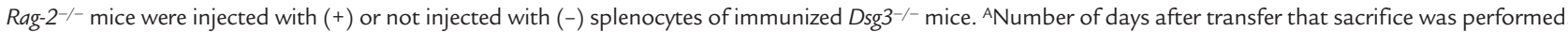
Number of Anti-Dsg3 IgG-producing B cells is shown per $10^{5}$ mononuclear cells in spleen, lymph nodes, bone marrow, and PBMC. 

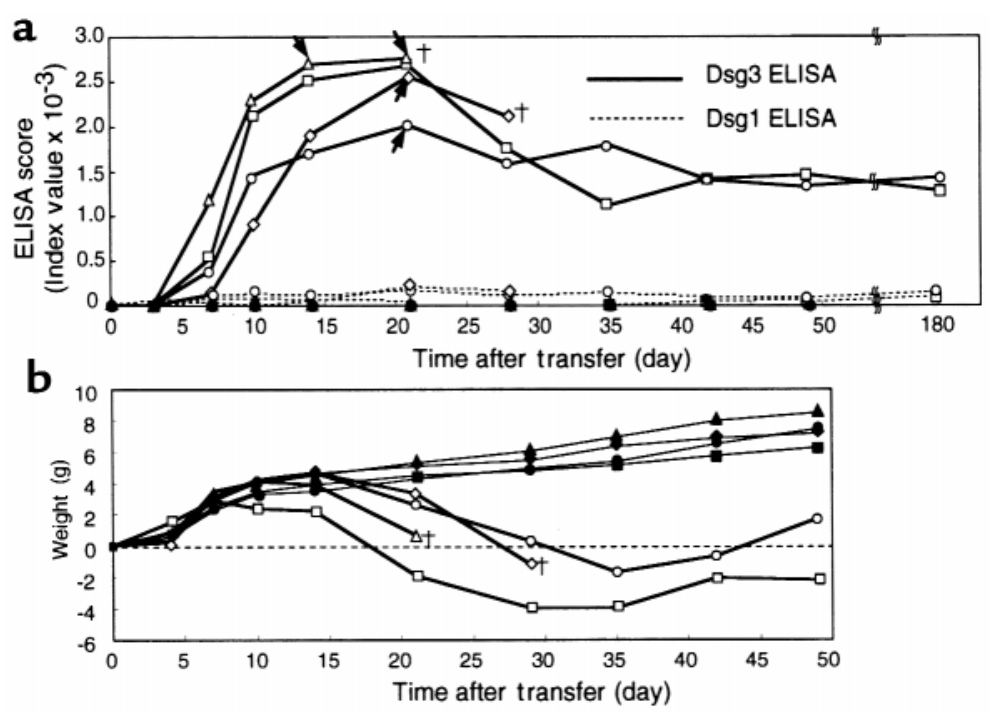

Figure 2

Stable production of anti-Dsg3 IgG in mice given immunized $D s g 3^{-/-}$splenocytes. Splenocytes were isolated from immunized Dsg3-/- or Dsg3 $3^{+/-}$mice and then transferred to Rag-2 $2^{-/-}$mice by intravenous injection into the tail vein. (a) Circulating anti-Dsg3 IgG was detected in the mice that received Dsg3-/- splenocytes (open symbols, solid line), but not in the recipients of $D s g 3^{+/-}$ splenocytes (solid symbols). The antibody production persisted for more than 6 months. There was no apparent reactivity against Dsg1 (dashed line). Arrows indicate the day when the hair loss phenotype became apparent. (b) Weight change in the recipient Rag-2-/mice is shown by plotting weight against time.tindicates the death of a mouse.

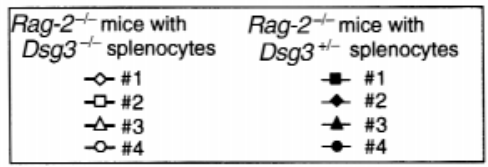

throughout the layers of living epithelium. In these mice, no IgG deposition was found in other tissues, including heart, lung, liver, kidney, stomach, and small and large intestines (data not shown). These IgG binding sites correspond to the known tissue distribution of Dsg3 $(5,20,21)$.

Histologic examination of the recipient mice revealed an intraepithelial loss of cell-cell adhesion just above the basal layers (i.e., suprabasilar acantholysis), in the buccal mucosa, hard palate (Figure $3 \mathrm{~g}$ ), oropharyngeal areas, and the upper part of the esophagus (Figure $3 \mathrm{~h}$ ). These are typical histologic findings in PV patients (Figure 3j). These oral erosions probably inhibited food intake, resulting in the weight loss. No significant infiltration of inflammatory cells was observed in the early stages of developing blisters (Figure 3, $g$ and h). In contrast, no phenotypic or pathologic changes were observed in Rag-2/- mice that received immunized $D s \mathrm{~s}^{+/-}$splenocytes (Figure 3 , e and i). There was no apparent sign of graft-versus-host disease in the skin or intestines in any of these mice given $D s g 3^{-/-}$or $D s g 3^{+/-}$splenocytes (data not shown).

All 18 mice receiving $D s g 3^{-/-}$splenocytes showed positive in vivo IgG deposition on keratinocyte cell surfaces, although 3 of them did not show apparent weight loss or histologic acantholysis (Table 2). The IgG titers in these 3 mice were significantly lower than those in the other 15 mice; peak index values were $(0.7 \pm 0.4) \times 10^{3}$ and $(2.4 \pm 0.9) \times 10^{3}$, respectively $(P<0.01)$. When mice with lower titers were boosted with $\mathrm{rDsg} 3$, the titers rose and the mice developed the PV characteristics (data not shown). Therefore, the appearance of the PV characteristics might generally be correlated with the antibody titer. These results indicate that the Rag-2-/- mice given immunized $D s g 3^{3^{-/}}$splenocytes developed clinical, histologic, and immunopathologic characteristics similar to those of $\mathrm{PV}$ patients.

Rag-2-/- mice with immunized Dsg $3^{-/-}$splenocytes develop alopecia. Around 15-25 days after the adoptive transfer, we also observed patchy hair loss in the Rag- $2^{-/}$mice that received immunized $D s g 3^{--}$splenocytes (see arrows in Figure 2a; Figure 4, a and b), but not in those that received $D s g 3^{+/-}$splenocytes. Typically, the bald areas started as small spots and gradually enlarged peripherally over the next $2-3$ weeks. In some mice, new hair grew in the bald areas in a patchy pattern (Figure 4c), whereas in others, the bald areas remained in the same spot for more than 1 month. Some mice had diffuse hair loss with no discrete bald areas. This hair loss also persisted for more than 6 months. Skin biopsy showed intense IgG deposition on the cell surface of keratinocytes surrounding the telogen hair club (Figure 4d). Cleft formation was observed between the cells surrounding the tel-

Table 2

Summary of PV phenotype observed in Rag-2-/- recipient mice

\begin{tabular}{|c|c|c|c|c|c|c|c|c|}
\hline Rag-2-/- mice with & $\begin{array}{l}\text { Number } \\
\text { tested }\end{array}$ & $\begin{array}{l}\text { Positive } \\
\text { ELISA }^{\mathrm{A}}\end{array}$ & $\begin{array}{l}\text { Living cell } \\
\text { Staining }\end{array}$ & $\begin{array}{l}\text { Positive } \\
\text { DIFC }\end{array}$ & $\begin{array}{l}\text { Acantholysis } \\
\text { in histology }\end{array}$ & $\begin{array}{l}\text { Weight loss } \\
\text { Phenotype }^{E}\end{array}$ & $\begin{array}{l}\text { Crusted erosive } \\
\text { Lesions }\end{array}$ & $\begin{array}{l}\text { Hair loss } \\
\text { Phenotype }\end{array}$ \\
\hline 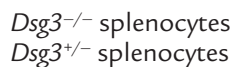 & $\begin{array}{l}18 \\
10\end{array}$ & $\begin{array}{c}18 \\
0\end{array}$ & $\begin{array}{c}18 \\
0\end{array}$ & $\begin{array}{c}18 \\
0\end{array}$ & $\begin{array}{c}15 \\
0\end{array}$ & $\begin{array}{c}15 \\
0\end{array}$ & $\begin{array}{l}6 \\
0\end{array}$ & $\begin{array}{c}13 \\
0\end{array}$ \\
\hline
\end{tabular}

Number of mice with positive finding for each category is shown. ${ }^{A}$ Circulating IgG was tested with ELISA against mouse rDsg3. ${ }^{B}$ Circulating IgG was determined by staining of living keratinocytes (PAM212). CIn vivo IgG deposition on keratinocyte cell surfaces was determined by direct immunofluorescence staining of oropharyngeal mucous membranes. DIntraepithelial blister formation (i.e. suprabasilar acantholysis) was determined by histologic examination of the oropharyngeal areas. EWeight loss greater than $2 \mathrm{~g}$ within a week was considered significant. 
ogen club and the basal layer of the outer root sheath epithelium (Figure 4e). The bald skin contained empty, dilated telogen hair follicles, consistent with the loss of the telogen hair (Figure 4f). These clinical and histologic findings of telogen hair loss are virtually identical to those observed in $D s g 3^{-/-}$mice, as are the findings of erosions on the oral mucous membrane and the skin (13, 22 ), confirming the specificity and pathogenicity of the anti-Dsg3 IgG produced in these mice.

\section{Discussion}

In this study, we adopted a unique approach to develop an active disease mouse model of pemphigus, by assuming that self-tolerance against Dsg3 is lost in Dsg3 $3^{-/-}$mice. Immunization with mouse $\mathrm{rDsg} 3$ was indeed successful in generating anti-Dsg3 IgG capable of binding native mouse Dsg3 on living keratinocytes in $D s g 3^{-/-}$mice, but not in $D s g 3^{+/-}$littermates. When splenocytes containing activated lymphocytes specific for mouse Dsg3 were adoptively transferred to Rag-2- $2^{-1}$ immunodeficient mice that expressed Dsg3, the Dsg3specific lymphocytes encountered the endogenous Dsg3, resulting in stable production of anti-Dsg3 IgG antibody for more than 6 months. The adoptive transfer of purified T cells and B cells from $D s g 3^{-/-}$mice was sufficient to produce such anti-Dsg3 IgG (M. Amagai et al., unpublished data), excluding the possible involvement of other types of cells - including antigenpresenting cells from $D s 3^{-/-}$mice - in this antibody production. In contrast, the endogenous Dsg3 did not stimulate lymphocytes from immunized $\mathrm{Dsg}^{+/-}$mice; no anti-Dsg3 IgG was produced in the mice receiving those lymphocytes. The persistent ongoing production

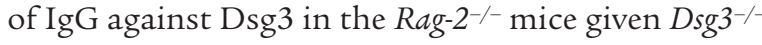
splenocytes up to 4 months after the transfer was confirmed by the ELISPOT assay. Furthermore, the antiDsg3 IgG was pathogenic, and the recipient $\mathrm{Rag}^{-2^{-/}}$ mice developed symptoms of PV, including erosions in the mucous membranes, which inhibited food intake with resultant weight loss, and scaly crusted erosions on traumatized skin, which resembles Nikolsky's sign (gentle rubbing of normal-appearing skin induces blisters). Consequently, this new approach has provided us with a novel active disease animal model of PV.

Clinically, PV is divided into a mucosal-dominant type and a mucocutaneous type. In mucosal-dominant $\mathrm{PV}$, oral erosions predominate, with limited skin involvement. In mucocutaneous PV, there are extensive skin lesions in addition to oral involvement. Recently, it was demonstrated that this clinical difference is defined by the anti-Dsg autoantibody profile (14, 23-25). Patients with mucosal-dominant PV have antiDsg3 IgG alone, whereas patients with mucocutaneous PV have anti-Dsg1 IgG in addition to anti-Dsg3 IgG. In the epidermis, Dsg1 is coexpressed with Dsg3. Therefore, anti-Dsg3 IgG alone is not able to cause skin blisters efficiently. On the other hand, in the mucous membrane Dsg3 is the dominant Dsg isotype, and antiDsg3 IgG alone is sufficient to cause oral erosions. In our model, $D s g 3^{-/-}$mice did not develop anti-Dsg1 IgG that was able to access the native Dsg1: no in vivo IgG deposition was observed in the immunized $D s g 3^{-/-}$mice that do express Dsg1, even after repeated immunization (data not shown). Furthermore, the Rag-2-/- mice
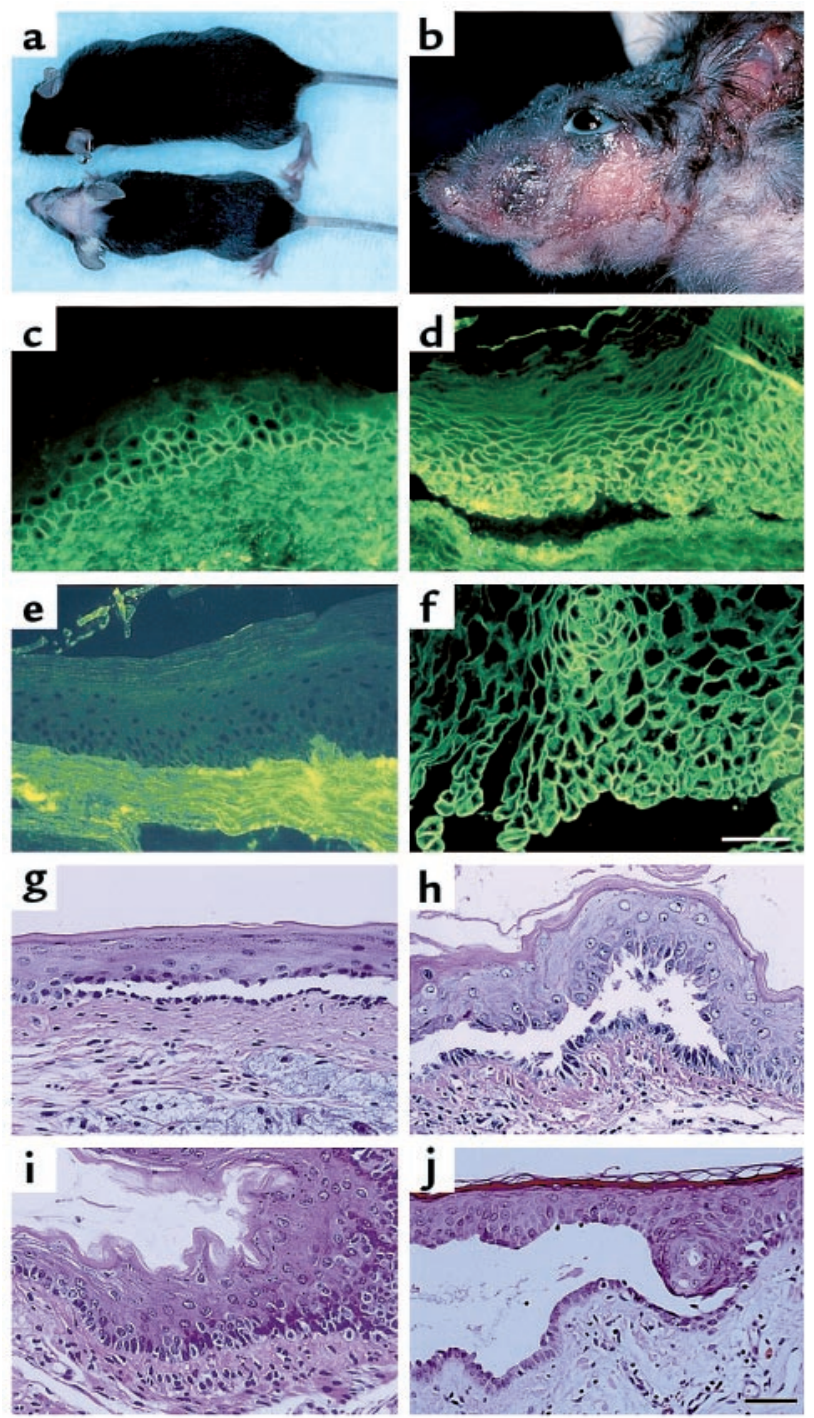

\section{Figure 3}

Rag-2-/- mice injected with immunized $D s g 3^{-/-}$splenocytes develop the PV phenotype. (a) Mice receiving Dsg3-/- splenocytes (bottom) were significantly smaller than mice given $D s g 3^{+/-}$splenocytes (top) 25-35 days after the adoptive transfer. (b) Some mice injected with Dsg3-/- splenocytes developed crusted erosions around the snout and cheeks, where mice normally scratch. (c-j) Histologic and immunopathologic examination of Rag- $2^{-/-}$recipient mice and patients with PV. In vivo IgG deposition on keratinocyte cell surfaces was observed in the skin (c, the skin around the snout) and mucous membranes ( $\mathbf{d}$, hard palate) of Rag-2-/mice given $D s g 3^{-/-}$splenocytes, just as is found in patients with PV (f, esophagus biopsy specimen). In contrast, there was no in vivo IgG deposition in the mice given $D s g 3^{+/-}$splenocytes (e, hard palate). The mice that received $D s g 3^{-/-}$splenocytes developed intraepithelial blisters just above the basal layers in mucosal epithelium ( $\mathbf{g}$, hard palate; $\mathbf{h}$, upper esophagus), which is a typical histologic finding in PV patients ( $\mathbf{j}$, skin). There was no apparent sign of acantholysis in the mice with $D s g 3^{+/-}$ splenocytes (i, upper esophagus). Bars $=50 \mu \mathrm{m}$. 

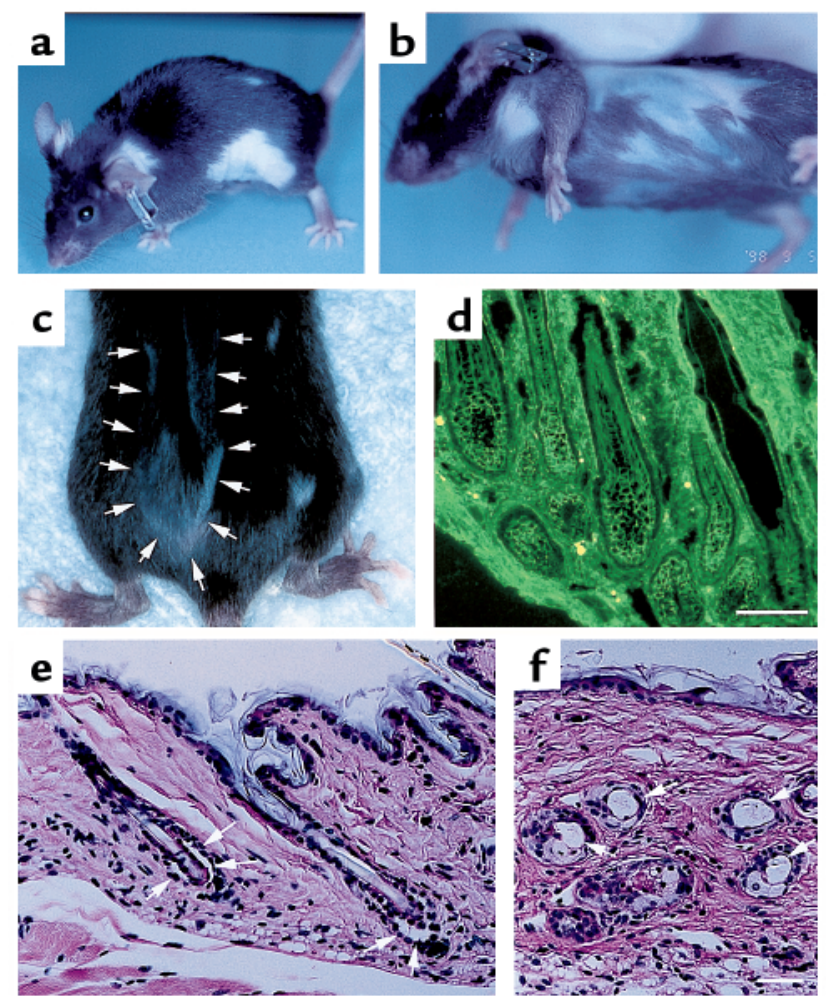

Figure 4

Hair loss phenotype of Rag-2-/- mice given splenocytes from immu-

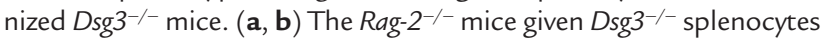
showed patchy hair loss, which first became apparent around day 15-25. (c) New hair growing in a bald area in a patchy pattern (arrows). (d) Intense in vivo IgG deposition was noticed on the cell surface of keratinocytes surrounding the telogen hair club. (e, f) Cleft formation between the cells surrounding the telogen club and the basal layer of the outer root sheath epithelium (e, arrows) and empty, dilated telogen hair follicles (f, arrows). Bars $=100 \mu \mathrm{m}$.

receiving $D s g 3^{-/-}$splenocytes did not develop any apparent anti-Dsg1 IgG with time (Figure 2a). This finding is consistent with our observation that the phenotypes of recipient mice were virtually identical to those of $D s g 3^{-/-}$mice. The IgG produced in these experiments was essentially specific for Dsg3, indicating that our PV model represents the mucosal-dominant type of PV.

Among the PV phenotypes that the recipient mice developed, in vivo IgG deposition on keratinocyte cell surfaces was found in all 18 mice injected with $D s g 3^{-/-}$ splenocytes (Table 2). This in vivo antibody binding to the target antigen is the first and essential step leading to the development of the PV phenotype. Acantholysis in the oral mucosa with resultant weight loss was observed in 15 of the 18 mice; the 3 remaining mice had lower titers of anti-Dsg3 IgG. Telogen hair loss was observed less frequently than was acantholysis or weight loss (Table 2). In mice, hair follicles in any particular area cycle in a synchronous fashion and enter the same stage at approximately the same time (26). This tendency becomes less evident as mice age. In addition, for a certain period mice have a normal coat, because hair regrows when the hair cycle enters the anagen stage (22).
Therefore, the hair loss phenotype might have been missed because some mice died or were sacrificed before the hair loss became apparent.

Previously, an in vivo experimental model of PV was developed by reconstituting severe combined immunodeficiency (SCID) mice with PBMC from patients with PV (27). In that model, lymphocytes from the patients produced a low titer of circulating anti-human Dsg3 IgG, but the spontaneous intraepidermal blisters associated with human IgG deposits were seen only rarely in the mouse skin. After grafting human skin on the SCID mice, PV-like blisters were observed in the grafted skin, although it remains to be determined whether inflammatory reactions due to possible histoincompatibility of human PBMC and skin contributed to the blister development in that model. Therefore, the mouse model developed in our study is the first solid active disease model of PV.

A major hurdle in developing animal models of autoimmune diseases has been overcoming self-tolerance. To create our model of disease, we circumvented this problem by immunizing autoantigen-knockout mice with the autoantigen, then transferring their splenocytes to $\operatorname{Rag}^{-2^{-/}}$mice that expressed the autoantigen. Although this model does not address the usual triggers of autoimmune diseases, it does provide a means to investigate the roles of $\mathrm{T}$ and $\mathrm{B}$ lymphocytes in perpetuating autoantibody production in the autoimmune response. In addition, this active animal model should be beneficial for evaluating various therapeutic strategies that could modulate the autoimmune response. Finally, because it is very easy to evaluate disease activity in this model through weight loss and hair loss, it can be used for efficient screening of various therapeutic interventions.

Our approach is widely applicable to various antibody-mediated and $\mathrm{T}$ cell-mediated autoimmune diseases (excluding cases where the relevant autoantigenknockout mice are embryonic lethal or show gross abnormalities in their immune systems). Furthermore, this approach provides another dimension in the use of knockout mice for study of the function of target molecules in vivo.

\section{Acknowledgments}

We thank John R. Stanley for reviewing the manuscript and for insightful discussion, and Tasuku Honjo for valuable discussion. We also thank John R. Stanley and Leonard D. Shultz for breeding pairs of $D s g 3^{-/-}$mice, Jouni Uitto for providing mouse Dsg3 cDNA, and Norihisa Matsuyoshi, John R. Stanley, Leena Pulkinen, and Jouni Uitto for providing mouse Dsg1 cDNA. Thanks also go to Akihiro Umezawa for preparing pathologic slides, Yoshiko Fujii for cell culture and for preparing recombinant baculoproteins, Minae Suzuki for immunofluorescence staining, and Ayumi Sakurai for animal care. This work was supported by Health Sciences Research Grants for Research on Specific Diseases from the Ministry of Health and Welfare of Japan 
(to M. Amagai), a Grant-in-Aid of Scientific Research (to M. Amagai, S. Koyasu, and T. Nishikawa), a Grantin-Aid of International Scientific Research from the Ministry of Education, Science, Sports and Culture of Japan (to T. Nishikawa), Keio Gijuku Academic Development Funds (to M. Amagai), and a grant from the Japan Society for the Promotion of Science (to S. Koyasu, JSPS-RFTF97L00701).

1. MacDonald, H.R. 1989. Mechanisms of immunological tolerance. Science. 246:982.

2. Goodnow, C.C. 1996. Balancing immunity and tolerance: deletion and tuning lymphocyte repertoires. Proc. Natl. Acad. Sci. USA. 93:2264-2271.

3. Bach, J.F., Koutouzov, S., and van Endert, P.M. 1998. Are there unique autoantigens triggering autoimmune diseases? Immunol. Rev. 164:139-155.

4. Stanley, J.R. 1998. Pemphigus. In Dermatology in general medicine. I.M Freedberg et al., editors. McGraw-Hill. New York, NY. 654-666.

5. Amagai, M., Klaus-Kovtun, V., and Stanley, J.R. 1991. Autoantibodies against a novel epithelial cadherin in pemphigus vulgaris, a disease of cell adhesion. Cell. 67:869-877.

6. Stanley, J.R. 1993. Cell adhesion molecules as targets of autoantibodies in pemphigus and pemphigoid, bullous diseases due to defective epidermal cell adhesion. Adv. Immunol. 53:291-325.

7. Amagai, M. 1996. Pemphigus: autoimmunity to epidermal cell adhesion molecules. Adv. Dermatol. 11:319-352.

8. Schiltz, J.R., and Michel, B. 1976. Production of epidermal acantholysis in normal human skin in vitro by the IgG fraction from pemphigus serum. J. Invest. Dermatol. 67:254-260.

9. Hashimoto, K., Shafran, K.M., Webber, P.S., Lazarus, G.S., and Singer, K.H. 1983. Anti-cell surface pemphigus autoantibody stimulates plasminogen activator activity of human epidermal cells. J. Exp. Med. 157:259-272.

10. Anhalt, G.J., Labib, R.S., Voorhees, J.J., Beals, T.F., and Diaz, L.A. 1982 Induction of pemphigus in neonatal mice by passive transfer of IgG from patients with the disease. N. Engl. J. Med. 306:1189-1196.

11. Amagai, M., Karpati, S., Prussick, R., Klaus-Kovtun, V., and Stanley, J.R. 1992. Autoantibodies against the amino-terminal cadherin-like binding domain of pemphigus vulgaris antigen are pathogenic. J. Clin. Invest. 90:919-926.

12. Amagai, M., Nishikawa, T., Nousari, H.C., Anhalt, G.J., and Hashimoto, T. 1998. Antibodies against desmoglein 3 (pemphigus vulgaris antigen) are present in sera from patients with paraneoplastic pemphigus and cause acantholysis in vivo in neonatal mice. J. Clin. Invest. 102:775-782.
13. Koch, P.J., et al. 1997. Targeted disruption of the pemphigus vulgaris antigen (desmoglein 3 ) gene in mice causes loss of keratinocyte cell adhesion with a phenotype similar to pemphigus vulgaris. J. Cell Biol. 137:1091-1102.

14. Ishii, K., et al. 1997. Characterization of autoantibodies in pemphigus using antigen-specific ELISAs with baculovirus expressed recombinant desmogleins. J. Immunol. 159:2010-2017.

15. Amagai, M., Hashimoto, T., Shimizu, N., and Nishikawa, T. 1994 Absorption of pathogenic autoantibodies by the extracellular domain of pemphigus vulgaris antigen (Dsg3) produced by baculovirus. J. Clin. Invest. 94:59-67.

16. Amagai, M., Hashimoto, T., Green, K.J., Shimizu, N., and Nishikawa, T. 1995. Antigen-specific immunoadsorption of pathogenic autoantibodies in pemphigus foliaceus. J. Invest. Dermatol. 104:895-901.

17. Schulz, R.J., Parkes, A., Mizoguchi, E., Bhan, A.K., and Koyasu, S. 1996. Development of CD4-CD8 ${ }^{-} \alpha \beta \mathrm{TCR}^{+} \mathrm{NK} 1.1^{+} \mathrm{T}$ lymphocytes: thymic selection by self antigen. J. Immunol. 157:4379-4389.

18. Amagai, M., et al. 1999. Usefulness of enzyme-linked immunosorbent assay (ELISA) using recombinant desmogleins 1 and 3 for serodiagnosis of pemphigus. Br. J. Dermatol. 140:351-357.

19. Yuspa, S.H., Hawley-Nelson, P., Koehler, B., and Stanley, J.R. 1980. A survey of transformation markers in differentiating epidermal cell lines in culture. Cancer Res. 40:4694-4703.

20. Schafer, S., Koch, P.J., and Franke, W.W. 1994. Identification of the ubiquitous human desmoglein, Dsg2, and the expression catalogue of the desmoglein subfamily of desmosomal cadherins. Exp. Cell Res. 211:391-399.

21. Amagai, M., Koch, P.J., Nishikawa, T., and Stanley, J.R. 1996. Pemphigus vulgaris antigen (Desmoglein 3 ) is localized in the lower epidermis, the site of blister formation in patients. J. Invest. Dermatol. 106:351-355.

22. Koch, P.J., et al. 1998. Desmoglein 3 anchors telogen hair in the follicle. J. Cell Sci. 111:2529-2537.

23. Ding, X., et al. 1997. Mucosal and mucocutaneous (generalized) pemphigus vulgaris show distinct autoantibody profiles. J. Invest. Dermatol. 109:592-596.

24. Amagai, M., Tsunoda, K., Zillikens, D., Nagai, T., and Nishikawa, T. 1999. The clinical phenotype of pemphigus is defined by the anti-desmoglein autoantibody profile. J. Am. Acad. Dermatol. 40:167-170.

25. Mahoney, M.G., et al. 1999. Explanation for the clinical and microscopic localization of lesions in pemphigus foliaceus and vulgaris. J. Clin. Invest. 103:461-468.

26. Dry, F.W. 1926. The coat of the mouse (Mus musculus). J. Genet 16:287-340.

27. Juhasz, I., Lazarus, G.S., Murphy, G.F., Shih, I.M., and Herlyn, M. 1993. Development of pemphigus vulgaris-like lesions in severe combined immunodeficiency disease mice reconstituted with lymphocytes from patients. J. Clin. Invest. 92:2401-2407. 